\title{
Darmreinigung
}

\section{Natriumpicosulfat als Split Dose zugelassen}

- Das Präparat CitraFleet ${ }^{ø}$ wird zur Darmreinigung eingesetzt, wenn eine diagnostische Untersuchung ansteht, die nur bei einem gut gereinigten Darm sinnvoll durchgeführt werden kann. Beispiele sind Koloskopie- und Röntgenuntersuchungen. Seit Dezember 2015 hat das Mittel nun die sogenannte SplitDose-Zulassung. Bei diesem Dosisregime wird ein Beutel am Abend vor der Untersuchung, der zweite Beutel dann am Morgen des eigentlichen Untersuchungstags eingenommen.

Bei den kleinvolumigen Natriumpicosulfat-Lösungen ist dies ein Novum in der Anwendung. Vergleichbare an- dere Produkte sind für eine auf zwei Tage aufgeteilte Einnahme nicht zugelassen. Das Split-Regime zeigt gegenüber der klassischen Einnahme eine klinisch signifikant verbesserte Reinigungsleistung. Die Einnahme von CitraFleet ${ }^{\circ}$ kann aber nach wie vor auf die klassische Art erfolgen, bei der ein Beutel am Nachmittag und ein zweiter am Abend vor der Untersuchung eingenommen werden. Dies ist empfehlenswert, wenn die Untersuchung am frühen Morgen stattfindet. Die neue Option sorgt für mehr Flexibilität in der Planung.

Red.

- Nach Informationen von Recordati

\section{Kurz notiert}

Patienteninformationen zu Cholesterin $\rightarrow$ Mit einer neuen will die Firma Sanofi über erhöhte Cholesterinwerte und die damit verbundenen gesundheitlichen Risiken aufkären. Unter www.cholesterin-unter-kontrolle.de finden Patienten, ihre Angehörigen und andere Interessierte zahlreiche Informationen.

Das „Herzstück“ der Seite ist der Dokumentarfilm „Heart Felt - True Stories of Life in the Shadow of High LDL Cholesterol“ der amerikanischen Filmemacherin Cynthia Wade, die für andere Dokumentationen bereits mit einem Oscar ausgezeichnet wurde. Eindrucksvoll erzählt der Film die Geschichten von Patienten aus fünf Ländern, die mit den teilweise gravierenden Folgen erhöhter Cholesterinwerte leben müssen. „Heart Felt" stellt dabei auch Patienten aus Deutschland und ihren Arzt vor.

Einen zusätzlichen Schwerpunkt der Seite bildet die Familiäre Hypercholesterinämie.

Red.

- Nach Informationen von Sanofi-Aventis

\section{Ginkgo-Extrakt in S3-Leitlinie aufgenommen}

_ Unter Federführung der Deutschen Gesellschaft für Psychiatrie und Psychotherapie, Psychosomatik und Nervenheilkunde (DGPPN) sowie der Deutschen Gesellschaft für Neurologie (DGN) wurde die Leitlinie „Demenzen“ überarbeitet. Die überarbeitete Leitlinie der höchsten methodischen Qualitätsstufe (S3) formuliert über 90 Schlüsselempfehlungen zu prioritären Versorgungsfragen.

Eine wichtige Neuerung: Die Verwendung des Ginkgo biloba Extrakts EGb $761^{\circ}$ wurde in die Reihe der Behandlungsmöglichkeiten aufgenommen, die bei leichter bis mittelschwerer De-

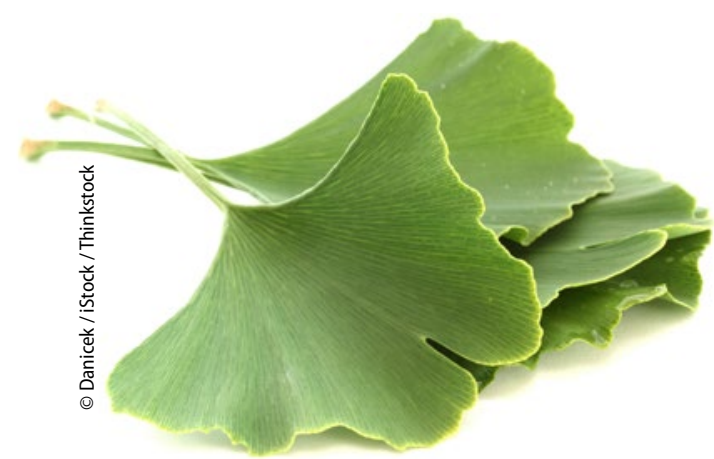

men. Die von den beteiligten Experten einstimmig gegebene Empfehlung beruht auf neuen Untersuchungen zur Verbesserung der geistigen Leistungsfähigkeit.

Die Empfehlung betrifft ausdrücklich nicht alle Ginkgo-Präparate, sondern den Spezialextrakt EGb $761^{\circ}$ (Tebonin $^{\circ}$ ), mit dem die neuen klinischen Studien durchgeführt wurden. Je nach Herstellungsprozess können sich verschiedene Ginkgo-Produkte stark unterscheiden. Daher sind sie nicht untereinander austauschbar [Deutsche Pharmazeutische Gesellschaft: Leitlinie Gute Substitutionspraxis. 2014]. Untersucht wurde eine Dosierung von einmal täglich einer Filmtablette mit $240 \mathrm{mg}$. Nur bei dauerhafter Einnahme konnte die Wirkung nachgewiesen werden.

Bei Patienten bessert die regelmäßige Einnahme Konzentration, Gedächtnis und seelische Veränderungen wie Reizbarkeit oder Niedergeschlagenheit. Sie können damit den Alltag wieder besser bewältigen.

Red.

- S3-Leitlinie „Demenzen“, AWMF-Registernr. 038-013

\section{Kurz notiert}

Neue Opioid-Pflaster $\rightarrow$ Mit dem Vier-Tage Pflaster Bupre - 1 A Pharma ${ }^{\circledR}$ und der SiebenTage-Variante Bupre - 1 A Pharma ${ }^{\circledR} 7$ Tage kommen zwei neue transdermale Opioid-Pflaster auf den Markt. Der Wirkstoff Buprenorphin zählt zu den stark wirksamen Opioiden und hat Betäubungsmittelstatus.

Das Sieben-Tage-Pflaster ist zugelassen für die Behandlung von mittelstarken, nichtmalignen Schmerzen, wenn ein Opioid zum Erreichen einer adäquaten Analgesie notwendig ist. Es ist ebenso wie das Erstanbieterprodukt Norspan ${ }^{\circledast}$ in den Wirkstärken 5, 10 und $20 \mu \mathrm{g} /$ Stunde sowie in den Packungsgrößen 4, 8 und 12 Stück erhältlich. Zusätzlich bietet $1 \mathrm{~A}$ Pharma mit 15 $\mu \mathrm{g} /$ Stunde eine weitere Stärke an.

Das Vier-Tage-Pflaster ist indikationsgleich zu Transtec $\mathrm{PRO}^{\oplus}$. Es ist zur Anwendung bei mäßig starken bis starken Tumorschmerzen und starken Schmerzen bei ungenügender Wirksamkeit nicht-opioider Schmerzmittel angezeigt. Das Produkt steht in den Wirkstärken 35, 52,5 und $70 \mu \mathrm{g} /$ Stunde sowie in den Packungsgrößen 4, $5,8,10,16$ und 20 Stück zur Verfügung.

Red.

- Nach Informationen von 1 A Pharma 